\title{
UTILIZAÇÃO DE MISTURAS DE ÓLEO DE SOJA REUTILIZADO COM ÓLEO DIESEL EM UM MOTOR ESTACIONÁRIO
}

Maitê Edite Sousa Maronhas ${ }^{1}$, Haroldo Carlos Fernandes ${ }^{2}$, Wagner da Cunha Siqueira ${ }^{3}$, Lara Santana Fernandes ${ }^{4}$

\section{RESUMO}

Em todo o país, parte da população, em especial da zona rural, tem pouco ou nenhum acesso aos serviços de energia elétrica. Por isso, o uso de energia renovável para produção de energia elétrica na própria propriedade torna-se uma alternativa interessante. Neste trabalho, objetivou-se analisar a reutilização de óleo de soja para o acionamento de motores estacionários, sabendo-se que esse óleo, de outra forma, se tornaria resíduo e degradaria o meio ambiente, além de ser uma matéria-prima de baixo custo. Os ensaios foram realizados no Laboratório de Mecanização Agrícola, do Departamento de Engenharia Agrícola, da Universidade Federal de Viçosa, MG, utilizando-se um motor diesel estacionário marca Yanmar $\mathrm{NS}_{\mathrm{B}} 75$, com potência nominal de 5,8 kW a $2400 \mathrm{rpm}$, com injeção direta e refrigerado a água. Foram utilizados como combustíveis cinco misturas de óleo diesel (OD) e óleo de soja (OS) reutilizado, proveniente do restaurante universitário, nas proporções de $0-100 \%, 25-75 \%, 50-50 \%, 75-25 \%$ e $100-0 \%$, respectivamente. A potência e torque do motor foram maiores para as misturas de $75 \%$ OD e de $25 \%$ OS, e menores para $25 \%$ OD e $75 \%$ OS. O menor consumo horário deuse com a mistura de $25 \%$ OD e $75 \%$ OS e foi $15 \%$ menor para o diesel puro. Os valores encontrados justificam o uso de misturas de óleo diesel e óleo de soja reutilizado; porém, aspectos técnicos, em especial o desgaste do motor, devem ser avaliados após um longo período de funcionamento.

Palavras-Chave: Motor diesel, misturas de combustível, desempenho do motor

\section{EVALUATION OF A STATIONARY DIESEL ENGINE RUNNING ON MIXTURE OF USED SOYBEAN AND DIESEL OIL}

\begin{abstract}
Part of the Brazilian population especially, in the rural areas, has little or no access to electricity. Thus use of renewable energy for on site production of electricity is an appropriate alternative. The objective of this study was to analyze the use of low-cost used soybean oil (SO) to run stationary engines, because otherwise it goes waste and causes pollution. The study was done in the Laboratory of Agricultural Mechanization of the Department of Agricultural Engineering, at of the Federal University of Viçosa. The water cooled stationary diesel engine, Yanmar NSB 75, with direct injection, normal rated power of $5.8 \mathrm{~kW}$, at $2400 \mathrm{rpm}$, was used in the study. The SO was mixed with diesel oil (DO) in proportions of $0-100 \%, 25-75 \%, 50-50 \%, 75-25 \%$ and $100-0 \%$, respectively. The power and torque of the engine was higher with the use of $75 \% \mathrm{DO}$ and $25 \% \mathrm{SO}$ and low for $25 \% \mathrm{DO}$ and $75 \% \mathrm{SO}$. The hourly consumption was less with $25 \% \mathrm{DO}$ and $75 \%$ SO mixture, but $15 \%$ lower than for DO. The combined values suggest the possible use of DO and SO mixture, however technical aspects, especially engine wear and tear after a long operational period should be evaluated
\end{abstract}

Keywords: Diesel engine, fuel mixtures, engine performance.

\section{Recebido para publicação em 27/07/2009. Aprovado em 20/01/2010}

1- Engenheira Agrícola e Ambiental, Universidade Federal de Viçosa -MG - maronhas@gmail.com.

2- Professor Associado, Universidade Federal de Viçosa - MG - haroldo@ufv.br.

3- Doutorando em Mecanização Agrícola, Universidade Federal de Viçosa - MG - wagnercsiqueira@yahoo.com.br.

4- Graduanda em Engenharia de Alimentos, Universidade Federal de Viçosa - MG - Lara.fernandes@ufv.br 


\section{INTRODUÇÃO}

Conforme o Ministério de Minas e Energia (2006), no Norte do país, $62,5 \%$ da população rural (cerca de 2,6 milhões de pessoas); no Nordeste, $39,3 \%$ dos moradores da área rural (cerca de 5,8 milhões de pessoas); no Centro-Oeste, 27,6\% (cerca de $367 \mathrm{mil}$ pessoas); no Sudeste, $11,9 \%$ (cerca de 807 mil) e, no Sul, 8,2\% (cerca de 484 mil pessoas) não tem acesso aos serviços de energia elétrica Para minimizar esse problema, uma estratégia a ser adotada é o uso de energia renovável para a produção de energia elétrica na própria propriedade.

A utilização do óleo vegetal, como combustível alternativo ao óleo diesel, pode ser realizada usando o biodiesel ou da alimentando o do motor com óleo vegetal bruto ou in natura.

O biodiesel é o produto resultante do processo químico denominado transesterificação, em que óleos vegetais, animais ou minerais são acrescentados a uma mistura de substância catalisadora e álcool. Após a completa reação, é obtido o éster, que recebe o nome de biodiesel, tendo a glicerina como subproduto.

Em contrapartida, o óleo vegetal pode ser utilizado in natura, sendo extraído do grão ou amêndoa por meio de processos físicos.

Além do óleo extraído de grãos, outra alternativa é a utilização de óleos residuais de uso doméstico, de restaurantes e de indústrias. O óleo usado tornase um resíduo indesejado e sua reciclagem, como biocombustível alternativo, não só retiraria do meio ambiente um poluente, mas também permitiria a geração de uma fonte alternativa de energia, atendendo, assim, a duas necessidades básicas de uma só vez (COSTA NETO et al., 1999).

A fim de atender à produção de óleos vegetais, muitas culturas poderão ser utilizadas, de acordo com as condições regionais, destacando-se aquelas que já são exploradas comercialmente, como amendoim, soja, milho, dendê, girassol, canola e as de caráter regional, como mamona, andiroba, pequi, buriti, inajá, carnaúba, pinhão-manso, entre outras.

As vantagens da utilização de óleo vegetal são inúmeras, perante os combustíveis oriundos do petróleo. As emissões de um motor acionado com óleo vegetal contêm $40 \%$ menos fuligem e são $75 \%$ mais puras em relação ao diesel fóssil. O óleo vegetal também propicia melhor lubrificação interna do motor. Na questão ambiental, não contribui para o efeito estufa, por ser oriundo de fontes renováveis.
Além disso, o óleo reciclado como combustível constitui forma de destinação final não poluidora para natureza (COSTA NETO et al., 1999).

De acordo com Kaltner (2004), os principais problemas percebidos no uso de óleos vegetais nos motores diesel são a dificuldade de partida a frio, em razão da elevada viscosidade dos óleos vegetais; a formação de gomas nos bicos injetores; o desgaste de componentes da bomba injetora, devido à acidez do óleo, e a formação de depósitos de carvão na câmara de combustão, nos cilindros e nas válvulas.

Segundo Sant'anna (2003), a ideia de aproveitar óleos vegetais para alimentar motores não é nova. Rudolf Diesel, inventor do motor a diesel, em 1893, propôs a utilização de óleo de amendoim como combustível; porém, o baixo custo do petróleo, naquela época, inibiu o projeto.

Para Moraes (1980), o óleo vegetal misturado ao óleo diesel, na forma in natura, na substituição do óleo diesel é alternativa viável, considerando-se, especialmente, os aspectos sociais.

Prateepchaikul e Apuchato (2003) estudaram o desempenho e durabilidade de motores Kubota, monocilíndricos, com injeção indireta, operados com rotação de $2200 \mathrm{rpm}$ e com $75 \%$ da carga nominal, alimentados com óleo diesel e óleo de dendê refinado, por um período de 2000 horas, em condições idênticas. Durante o experimento, os motores foram desmontados para análise de desgaste, logo após 50 horas de operação, e, posteriormente, a cada 500 horas, para análise dos componentes. Com isso, verificou-se que o consumo de óleo de dendê foi de 15 a 20\% maior. Ocorreu, apenas, diferença significativa no desgaste dos anéis de segmento, sendo que o maior desgaste foi encontrado no motor abastecido pelo óleo de dendê.

Ao avaliarem um motor MWM D229-3, de injeção direta, que acionava um trator Valmet modelo 68, alimentado com óleo diesel durante as 50 primeiras horas e, depois, exclusivamente, com óleo bruto de girassol, Mazeiro e Corrêa (2004) verificaram redução de 7,1 a $10,1 \%$ na potência da TDP e aumento de 13,9 a $16 \%$ no consumo específico. O teste de 200 horas foi interrompido com menos de 60 horas, por causa da elevação da temperatura do lubrificante. Os autores relataram, ainda, que a alta viscosidade do óleo bruto de girassol dificultava a pulverização dos bicos injetores, propiciando queima do combustível e, consequentemente, formação de depósitos nos bicos e cabeçotes, que levavam à redução do desempenho e da durabilidade do motor. 
Desta forma, neste trabalho teve como objetivouse avaliar o desempenho de um motor estacionário de ciclo diesel, utilizando misturas de óleo de soja reutilizado com óleo diesel.

\section{MATERIAL E MÉTODOS}

O presente trabalho foi conduzido nos Laboratórios de Qualidade da Água e de Mecanização Agrícola, do Departamento de Engenharia Agrícola, da Universidade Federal de Viçosa, MG, no período de 08/2007 a 07/2008.

Foram utilizados dois tipos de óleos, óleo diesel puro (OD) e óleo de soja reutilizado (OS), proveniente do restaurante universitário da Universidade Federal de Viçosa, nas seguintes misturas: 0\%OD e 100\%OS, $25 \%$ OD e $75 \%$ OS, $50 \%$ OD e $50 \%$ OS, $75 \%$ OD e $25 \%$ OS e $100 \%$ OD e $0 \%$ OS.

Para realizar sua limpeza, o óleo reutilizado foi armazenado em tanques, onde ficou em repouso por uma semana, para que os resíduos se acumulassem no fundo do reservatório. Após esse processo, o óleo foi retirado pela parte superior do tanque e coado.

O sal contido no óleo pode causar danos aos componentes do motor. Para retirá-lo, o óleo, já decantado, foi colocado em tanques e misturado com água, em uma proporção de $10 \%$. A água solubilizou os elementos quimicos contidos no óleo em uma semana de descanso. Para a retirada da água da mistura, apenas a separação física não foi suficiente. Assim sendo, o óleo foi novamente retirado pela parte superior dos tanques e fervido por dez minutos, para retirada da água residual.

$\mathrm{O}$ experimento foi conduzido no Delineamento Inteiramente Casualizado (DIC) e foram amostrados seis pontos. Para a confecção dos gráficos foi utilizado o software EXCEL 2003.

Para a determinação do consumo específico de combustível, é necessário o conhecimento das densidades das diferentes misturas utilizadas. Para isto, foram utilizados um béquer com capacidade de $100 \mathrm{~mL}$ e precisão de $1 \mathrm{~mL}$, um termômetro com variação de $-10^{\circ} \mathrm{C}$ a $210^{\circ} \mathrm{C}$, com precisão de $2^{\circ} \mathrm{C}$, e uma balança com capacidade para $1500 \mathrm{~g}$ e precisão de $0,01 \mathrm{~g}$.

$\mathrm{O}$ ensaio iniciou-se com a pesagem do óleo e a medição da temperatura da amostra. Esta era aquecida até que seu volume atingisse $55 \mathrm{~mL}$, tendo sua massa e sua temperatura medidas a cada $1 \mathrm{~mL}$ de variação do volume.

Utilizou-se um motor diesel estacionário, marca
Yanmar, modelo $\mathrm{NS}_{\mathrm{B}} 75$, com potência nominal de $5,8 \mathrm{~kW}$ a $2400 \mathrm{rpm}$, com injeção direta, refrigerado a água.

Os ensaios foram realizados nas dependências do Laboratório de Mecanização Agrícola, do Departamento de Engenharia Agrícola, da Universidade Federal de Viçosa.

O dinamômetro utilizado foi de rotação, do tipo transmissão montado em berço.

Como combustíveis, foram utilizadas as cinco misturas, descritas anteriormente. Para a realização dos ensaios, além do dinamômetro, também foram utilizados um fluxômetro de combustível, com capacidade para $250 \mathrm{~mL}$, um tacômetro digital de contato e um cronômetro digital. Os ensaios seguiram a norma NBR 5484 (1985) e para isto foram utilizadas temperaturas de bulbo úmido, de bulbo seco e dados de pressão barométrica, fornecidos pelo Instituto Nacional de Meteorologia (INMET).

O ensaio iniciava-se após dez minutos de funcionamento do motor com óleo diesel puro, para aquecimento; para as etapas de ensaio as misturas com algum percentual de óleo de soja seguiam-se mais dez minutos de funcionamento do motor com a mistura a ser testada. Para o encerramento, deixava-se também o motor funcionando por dez minutos com óleo diesel puro, para evitar formação de gomas e entupimentos.

Nos ensaios foi utilizado o tempo de coleta de dados de dois minutos, sendo coletados dados de rotação do motor e do dinamômetro, de carga do dinamômetro e de consumo de combustível. Foram realizadas três repetições para cada mistura de combustível.

As cargas aplicadas pelo dinamômetro foram variáveis, situando-se seus valores entre 33,3 e 97,8 N. $\mathrm{O}$ motor foi acoplado ao dinamômetro por meio de polias e correias tipo V.

Os dados foram coletados e usados para traçar os gráficos da potência, do torque, do consumo horário e do consumo específico de combustível, em função da rotação do motor.

Após coletados, os dados foram tabulados, com o auxílio do software EXCEL 2003, convertidos e corrigidos de acordo com a norma NBR 5484 (1985)

Para traçar os gráficos de potência em relação à rotação do motor, utilizou-se a equação:

$P=k F n 0,746$

em que,

$P=$ potência do motor, $\mathrm{kW}$;

$F=$ carga aplicada pelo dinamômetro, lbf;

$n=$ número de rotações por minuto, rpm; 
$k=$ constante do dinamômetro, 0,00025 .

Para traçar os gráficos de torque em relação à rotação do motor, utilizou-se a equação abaixo:

$$
T=\frac{60 P 1000}{2 \pi n}
$$

em que,

$T=$ torque do motor, $\mathrm{mN}$

$P=$ potência do motor, $\mathrm{kW}$

$n=$ número de rotações por minuto, rpm

Para traçar os gráficos de consumo horário em relação à rotação do motor, utilizou-se a equação:

$$
C h=\frac{60 C}{21000}
$$

em que,

$C h=$ consumo horário, $\mathrm{Lh}^{-1}$;

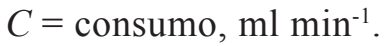

Para traçar os gráficos de consumo específico em relação à rotação do motor, utilizou-se a equação:

$$
C e=\frac{C h D}{P}
$$

em que,

$C e=$ consumo específico de combustível, $\mathrm{g} \mathrm{kW}^{-}$ ${ }^{1} \mathrm{~h}^{-1}$;

$C h=$ consumo horário, $\mathrm{L} \mathrm{h}^{-1}$;

$D=$ densidade do combustível, $\mathrm{g} \mathrm{L}^{-1}$;

$P=$ potência do motor, $\mathrm{kW}$.

\section{RESULTADOS E DISCUSSÃO}

Por meio da análise de regressão foram determinadas equações para estimar as densidades das misturas, potência, torque, consumo horário e consumo específico, que são apresentadas nos Quadros de 1 a 8, respectivamente. Os modelos foram lineares para densidade, potência, torque e consumo horário, e quadrático para o consumo específico. As estimativas das equações podem ser visualizadas nas Figuras de 1 a 5.

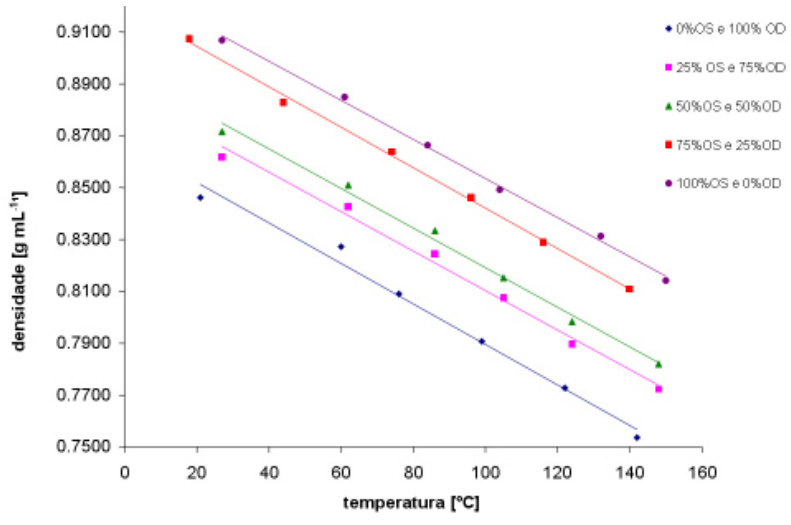

Figura 1. Comportamentodadensidade das misturas em função da variação da temperatura.

De acordo com a Figura 1, pode-se perceber que a densidade da mistura aumenta com o aumento da porcentagem de óleo de soja (OS) e diminui com o aumento da temperatura.

A mistura de maior densidade é a de $100 \%$ OS e $0 \%$ OD; e a de menor densidade é a de $0 \%$ OS e $100 \% \mathrm{OD}$, com uma diferença de $0,061 \mathrm{~g} \mathrm{~mL}^{-1}$, a $70{ }^{\circ} \mathrm{C}$.

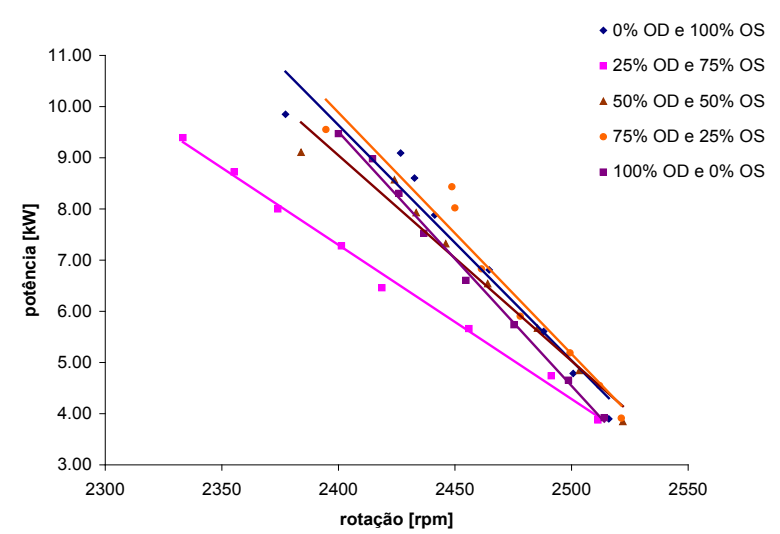

Figura 2. Comportamento da potência em função da rotação do motor.

Quadro 1. Equações geradas para a estimativa das densidades das misturas

\begin{tabular}{lll}
\hline Combustível & \multicolumn{1}{c}{ Equação } & $\boldsymbol{R}^{\mathbf{2}}$ \\
\hline $0 \%$ OD e 100\% OS & $\mathrm{D}_{100 \%}=-0.0008 \mathrm{~T}+0.9288$ & 0.9974 \\
$25 \%$ OD e 75\% OS & $\mathrm{D}_{75 \%}=-0.0008 \mathrm{~T}+0.9200$ & 0.9977 \\
$50 \%$ OD e 50\% OS & $\mathrm{D}_{50 \%}=-0.0008 \mathrm{~T}+0.8955$ & 0.9931 \\
$75 \%$ OD e 25\% OS & $\mathrm{D}_{25 \%}=-0.0008 \mathrm{~T}+0.8864$ & 0.9913 \\
$100 \%$ OD e 0\% OS & $\mathrm{D}_{0 \%}=-0.0008 \mathrm{~T}+0.8676$ & 0.9869 \\
\hline
\end{tabular}

em que,

D - Densidade da mistura

$\mathrm{T}$ - temperatura da mistura. 
Pode-se perceber que a mudança de combustível provocou variação na potência do motor, sendo a mistura de $25 \%$ OD e $75 \%$ OS a que obteve sempre menor potência, comparada com as demais.

Na Figura 2, as linhas de tendência correspondentes aos gráficos de $75 \%$ OD e $25 \%$ OS, $0 \%$ OD e $100 \%$ OS e $50 \%$ OD e $50 \%$ OS aproximam-se com o aumento da rotação.

Ocorreu uma queda de $24 \%$ na potência do motor quando o combustível utilizado passou de $75 \%$ OD e $25 \%$ OS para $25 \%$ OD e $75 \%$ OS.

Santos (2007) constatou que o aumento da potência e do torque no motor avaliado foi proporcional à adição de ORS na mistura com OD, trabalhando em rotações superiores a $2350 \mathrm{rpm}$.
A variação do torque do motor de acordo com as misturas pode ser verificada na Figura 3.

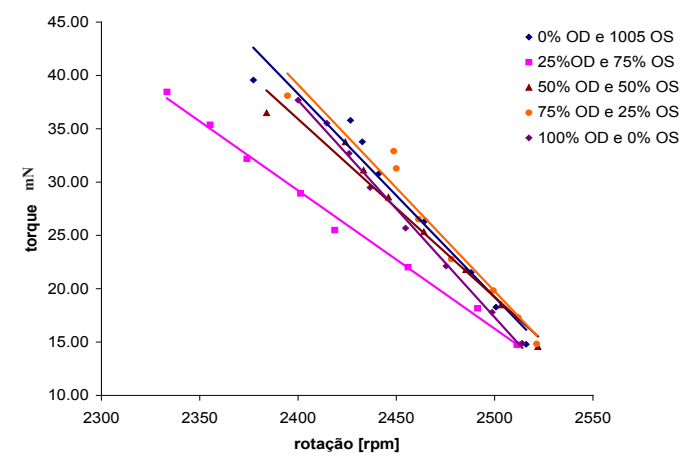

Figura 3. Comportamento do torque em função da rotação do motor.

Quadro 2. Equações geradas para a estimativa de potência

\begin{tabular}{clc}
\hline Combustível & \multicolumn{1}{c}{ Equação } & $\boldsymbol{R}^{\mathbf{2}}$ \\
\hline $0 \%$ OD e $100 \%$ OS & $\mathrm{P}_{100 \%}=-0.046 \mathrm{x}+120.06$ & 0.9496 \\
$25 \%$ OD e $75 \%$ OS & $\mathrm{P}_{75 \%}=-0.0301 \mathrm{x}+79.558$ & 0.9946 \\
$50 \%$ OD e $50 \%$ OS & $\mathrm{P}_{50 \%}=-0.0402 \mathrm{x}+105.47$ & 0.9691 \\
$75 \%$ OD e $25 \%$ OS & $\mathrm{P}_{25 \%}=-0.0472 \mathrm{x}+123.13$ & 0.9471 \\
$100 \%$ OD e $0 \%$ OS & $\mathrm{P}_{0 \%}=-0.0496 \mathrm{x}+128.64$ & 0.9958 \\
\hline
\end{tabular}

Quadro 3. Valores obtidos de potência $(\mathrm{kW})$ para a rotação de $2425 \mathrm{rpm}$

\begin{tabular}{cc}
\hline Combustível & Potência $[\boldsymbol{k} \boldsymbol{W}]$ \\
\hline $0 \%$ OD e $100 \%$ OS & 8,51 \\
$25 \%$ OD e $75 \%$ OS & 6,57 \\
$50 \%$ OD e $50 \%$ OS & 7,99 \\
$75 \%$ OD e $25 \%$ OS & 8,67 \\
$100 \%$ OD e $0 \%$ OS & 8,36 \\
\hline
\end{tabular}

em que,

$\mathrm{P}=$ Potência do motor, em kW para a porcentagem de óleo de soja (OS) da mistura;

$\mathrm{X}=$ rotação do motor, em rpm.

Quadro 4. Equações geradas para a estimativa de torque do motor

\begin{tabular}{ccc}
\hline Combustível & Equação & $\boldsymbol{R}^{\mathbf{a}}$ \\
\hline 0\%OD e $100 \%$ OS & $\mathrm{T}_{100 \%}=-0.1906 \mathrm{x}+495.68$ & 0.9602 \\
25\%OD e 75\%OS & $\mathrm{T}_{75 \%}=-0.1296 \mathrm{x}+340.20$ & 0.9939 \\
$50 \%$ OD e $50 \%$ OS & $\mathrm{T}_{50 \%}=-0.1669 \mathrm{x}+436.38$ & 0.9766 \\
$75 \%$ OD e $25 \%$ OS & $\mathrm{T}_{25 \%}=-0.1939 \mathrm{x}+504.43$ & 0.9561 \\
$100 \%$ OD e $0 \%$ OS & $\mathrm{T}_{0 \%}=-0.2035 \mathrm{x}+526.09$ & 0.9952 \\
\hline
\end{tabular}

em que,

$\mathrm{T}=$ Torque do motor, em $\mathrm{mN}$, para a porcentagem de óleo de soja (OS) da mistura;

$\mathrm{x}=$ rotação do motor, em rpm.

Quadro 5. Valores obtidos de torque do motor para a rotação de $2425 \mathrm{rpm}$

\begin{tabular}{cc}
\hline Combustível & Torque [m $\boldsymbol{N}]$ \\
\hline $0 \% \mathrm{OD}$ e $100 \% \mathrm{OS}$ & 33,48 \\
$25 \% \mathrm{OD}$ e $75 \% \mathrm{OS}$ & 25,92 \\
$50 \% \mathrm{OD}$ e $50 \% \mathrm{OS}$ & 31,65 \\
$75 \% \mathrm{OD}$ e $25 \% \mathrm{OS}$ & 34,22 \\
$100 \% \mathrm{OD}$ e $0 \% \mathrm{OS}$ & 32,60 \\
\hline
\end{tabular}


Pode-se perceber no Quadro 5, valores sempre menores quando o motor é alimentado com a mistura de $25 \%$ OD e $75 \%$ OS, e valores maiores quando ele é alimentando com a mistura de $75 \%$ OD e $25 \%$ OS, com tendência de se aproximarem com o aumento da rotação.

Ocorreu decréscimo de $24 \%$ no torque do motor quando o combustível utilizado passou de $75 \%$ OD e $25 \%$ OS para $25 \%$ OD e $75 \%$ OS.

Machado (2003), avaliando o desempenho de diferentes composições de óleo de soja com óleo diesel em um motor estacionário, verificou um maior torque, $37 \mathrm{mN}$, para a mistura de $70 \%$ de óleo de soja com $30 \%$ de óleo diesel.

Pode-se perceber, na figura 4, a variação do consumo horário de acordo com a mistura utilizada. A mistura de $25 \%$ OD e $75 \%$ OS apresentou um consumo horário menor do que as outras, e a mistura de $0 \%$ OD e $100 \%$ OS apresenta um consumo horário acima das outras misturas, até aproximadamente $2450 \mathrm{rpm}$, quando os gráficos das misturas tendem a se encontrar.

$\mathrm{O}$ maior consumo horário ocorreu quando o motor foi alimentando com a mistura de $100 \%$ OD e $0 \%$ OS; o menor, com a mistura de $25 \%$ OD e $75 \%$ OS, com uma variação de $15 \%$.

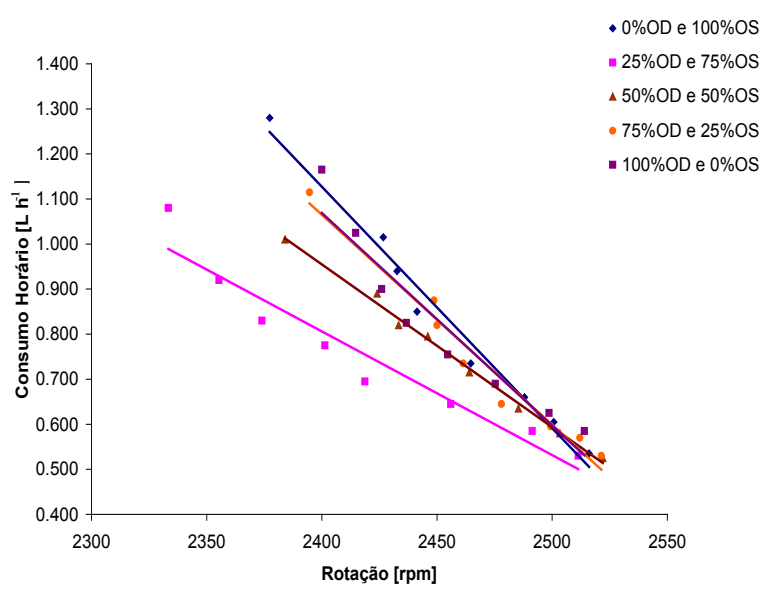

Figura 4. Comportamento do consumo horário em função da rotação do motor.

Quadro 6. Equações geradas para a estimativa de consumo horário do motor

\begin{tabular}{ccc}
\hline Combustível & Equação & $\boldsymbol{R}^{2}$ \\
\hline $0 \%$ OD e 100\% OS & $\mathrm{Ch}_{100 \%}=-0.0054 \mathrm{x}+13.986$ & 0.9801 \\
$25 \%$ OD e 75\% OS & $\mathrm{Ch}_{75 \%}=-0.0027 \mathrm{x}+7.3939$ & 0.9285 \\
$50 \%$ OD e 50\% OS & $\mathrm{Ch}_{50 \%}=-0.0036 \mathrm{x}+9.6266$ & 0.9943 \\
$75 \%$ OD e 25\% OS & $\mathrm{Ch}_{25 \%}=-0.0047 \mathrm{x}+12.254$ & 0.9665 \\
$100 \%$ OD e 0\% OS & $\mathrm{Ch}_{0 \%}=-0.0047 \mathrm{x}+12.391$ & 0.9145 \\
\hline
\end{tabular}

em que,

$\mathrm{Ch}=$ Consumo específico do motor $\mathrm{em} \mathrm{L} \mathrm{h}^{-1}$ para a porcentagem de óleo de soja (OS) da mistura;

$\mathrm{x}=$ rotação do motor em rpm.

Quadro 7. Valores obtidos de consumo horário para a rotação de $2425 \mathrm{rpm}$

\begin{tabular}{cc}
\hline Combustível & Consumo Horário [L $\left.\boldsymbol{h}^{-1}\right]$ \\
\hline $0 \%$ OD e $100 \%$ OS & 0,891 \\
$25 \%$ OD e $75 \%$ OS & 0,846 \\
$50 \%$ OD e $50 \%$ OS & 0,897 \\
$75 \%$ OD e $25 \%$ OS & 0,857 \\
$100 \%$ OD e $0 \%$ OS & 0,994 \\
\hline
\end{tabular}

Quadro 8. Equações geradas para a estimativa de consumo específico do motor

\begin{tabular}{lll}
\hline Combustível & Equação & $\boldsymbol{R}^{2}$ \\
\hline $0 \%$ OD e $100 \%$ OS & $\mathrm{CE}_{100 \%}=0.0049 \mathrm{x}^{2}-23.951 \mathrm{x}+29373$ & 0.9906 \\
$25 \%$ OD e $75 \%$ OS & $\mathrm{CE}_{75 \%}=0.0019 \mathrm{x}^{2}-9.2114 \mathrm{x}+11119$ & 0.9648 \\
$50 \%$ OD e $50 \%$ OS & $\mathrm{CE}_{50 \%}=0.0029 \mathrm{x}^{2}-14.322 \mathrm{x}+17489$ & 0.9496 \\
$75 \%$ OD e $25 \%$ OS & $\mathrm{CE}_{25 \%}=0.0045 \mathrm{x}^{2}-21.84 \mathrm{x}+26805$ & 0.9677 \\
$100 \%$ OD e 0\% OS & $\mathrm{CE}_{0 \%}=0.006 \mathrm{x}^{2}-29.441 \mathrm{x}+36009$ & 0.9819 \\
\hline
\end{tabular}

em que,

$\mathrm{Ce}=$ Consumo específico do motor em $\mathrm{g} \mathrm{kW}^{-1} \mathrm{~h}^{-1}$ para a porcentagem de óleo soja (OS) da mistura; $\mathrm{x}=$ rotação do motor em rpm. 


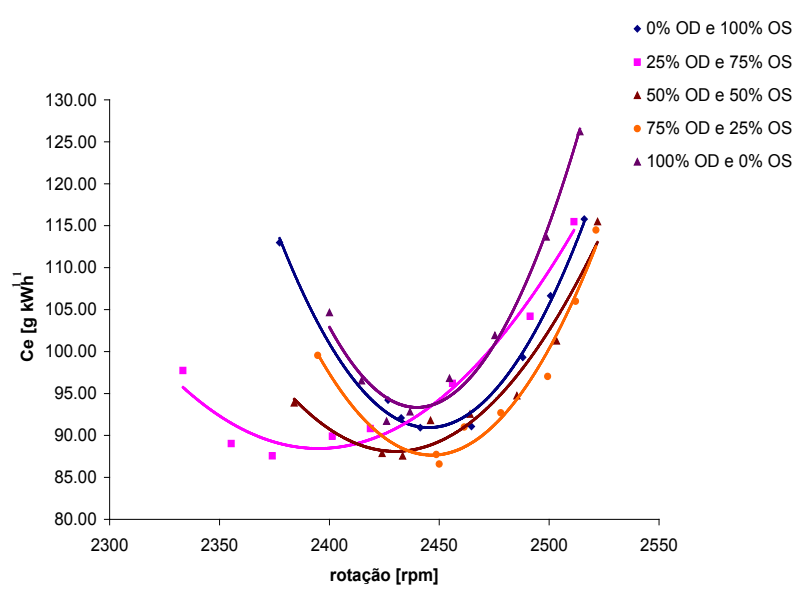

Figura 5. Comportamento do consumo específico em função da rotação do motor.

Pode-se perceber, na figura 5, que a mistura $100 \%$ OD e $0 \%$ OS tem o consumo específico sempre maior, quando comparada com as outras, e a mistura $75 \%$ OD e $25 \%$ OS apresenta menor consumo específico à rotação próxima de $2450 \mathrm{rpm}$. A mistura $50 \% \mathrm{OD}$ e $50 \% \mathrm{OS}$ apresenta menor consumo específico, entre 2400 e $2450 \mathrm{rpm}$, e a mistura de $25 \%$ OD e $75 \%$ OS apresenta menor consumo específico, próximo a $2400 \mathrm{rpm}$.

Santos (2007), trabalhando com óleo de soja refinado (ORS) em motores diesel de baixa potência, verificou maior consumo específico para as misturas ORS com diesel, em comparação com óleo diesel. Entretanto, em rotações superiores a $2350 \mathrm{rpm}$, as misturas demonstraram um menor consumo com relação ao óleo diesel.

\section{CONCLUSÕES}

Pode-se concluir, de acordo com os resultados obtidos neste trabalho, que:

- As densidades das misturas aumentam com o aumento da porcentagem de óleo de soja e diminuem com o aumento da temperatura;

- A potência e o torque do motor apresentaramse maiores para a mistura de $75 \%$ OD e $25 \%$ OS, acompanhados de um menor consumo específico. Para potência e torque esse aumento foi $24 \%$ maior em ambos, quando comparado com a mistura de menor potência e torque, que foi a de $25 \%$ OD e $75 \%$ OS;

- Para o consumo horário obteve-se $15 \%$ de variação entre o maior, que ocorre com a mistura de $100 \%$ OD e $0 \%$ OS, e o menor, que ocorre com a mistura de $25 \%$ OD e $75 \%$ OS;

- A mistura que atingiu o menor consumo específico foi a de $75 \%$ OD e $25 \%$ OS próximo à rotação de $2450 \mathrm{rpm}$;

- O óleo de soja reutilizado demonstrou ser um combustível eficiente e viável para ser utilizado em motores estacionários, desde que misturado em proporções menores do que o óleo diesel;

- São necessários estudos referentes ao desgaste do motor para utilização de misturas de óleo diesel e óleo de soja reutilizado, após um longo período de funcionamento do motor.

\section{REFERÊNCIAS BIBLIOGRÁFICAS}

ASSOCIAÇÃO BRASILEIRA DE NORMAS TÉCNICAS. NBR 5484: motores alternativos de combustão interna de ignição por compressão (Diesel) ou ignição por centelha (Otto) de velocidade angular variável - ensaio - Método de ensaio. Rio de Janeiro, $1985.8 \mathrm{p}$.

COSTA NETO, P. R.; ROSSI, L. F. S; ZAGONEL, G. F; RAMOS, L. P. Produção de biocombustível alternativo ao óleo diesel através da transesterificação de óleo de soja usado em frituras. 1999. Disponível em: < www.scielo.br/pdf/qn/v23n4/2654.pdf. $>$. Acesso em: 30 nov. 2007.

KALTNER, F. J. Geração de energia elétrica em pequenas comunidades da Amazônia, utilizando óleo vegetal "in natura" de espécies nativas como combustível em motores diesel. SAEX 2004. Módulo 1-Biomassa. P 9.

MACHADO, P. R. M. Avaliação de Desempenho do Óleo de Soja como Combustível para Motores Diesel. 2003. 212 f. Dissertação (Mestrado em Mecanização Agrícola)- Universidade Federal de Santa Maria, Santa Maria, RS. 2003

MAZIEIRO, J.V.G.; CORRÊA, I.M. Biocombustível em xeque. Revista Cultivar Máquinas. N. 32, p. 6-9. julh. 2004.

MINISTÉRIO DE MINAS E ENERGIA - MME, Balanço Energético Nacional. Análise Energética 
Brasileira - período 1970 a 2005. Brasília. 2006. Disponível em: $<$ www.mme.gov.br $>$. Acesso em: 05 abr. 2007.

MORAES, J. R. Manual dos óleos vegetais e suas possibilidades energéticas. CNI. 1980. 78p.

PRATEEPCHAIKUL, G; APICHATO, T.; Palm oil as a fuel for agricultural diesel engines: Comparative testing against diesel oil. Songklanakarin J. Sci. Technol., V.25, n.3, p. 317-326, 2003.

SANT'ANNA, J. P. Biodiesel alimenta motor da economia. Revista Química e Derivados, v. 414, abril de 2003. Disponível em: $<$ www.quimica.com. br.>. Acesso em: 29 abr. 2004. 